\title{
Singular Random Signals
}

\author{
Bernard Picinbono, Fellow, IEEE, and Jean-Yves Tourneret
}

\begin{abstract}
Singular random signals are characterized by the fact that their values at each time are singular random variables, which means that their distribution functions are continuous but with a derivative almost everywhere equal to zero. Such random variables are usually considered as without interest in engineering or signal processing problems. The purpose of this paper is to show that very simple signals can be singular. This is especially the case for autoregressive moving average (ARMA) signals defined by white noise taking only discrete values and filters with poles located in a circle of singularity introduced in this paper. After giving the origin of singularity and analyzing its relationships with fractal properties, various simulations highlighting this structure will be presented.
\end{abstract}

Index Terms-ARMA models, fractals, stochastic signals.

\section{INTRODUCTION}

A random variable (RV) $x$ is said to be singular if its distribution function (DF) $F_{x}(\xi)$ is continuous but with a derivative almost everywhere equal to zero. Thus, there is neither a probability density function (PDF) nor a probability mass at some points. Similarly, a discrete-time random signal $x[k], k$ integer is singular if, for each $k$, the $\mathrm{RV} x[k]$ is singular.

The existence of singular RVs is noted in more mathematically oriented books [1, p. 9] [2]. In the signal processing community, singular RVs are often considered as mathematical curiosities either ignored, as in [3] and [4], or presented with the comment that they are not of any practical interest [5].

However, it is shown in [2] that if $w_{k}$ is a symmetric Bernoulli white noise (SBWN), which means a sequence of independent and identically distributed (IID) RVs taking only the values \pm 1 with the same probabilities, then the $\mathrm{RV} x=\sum_{k=0}^{\infty} a^{k} w_{k}$ is singular for $a<1 / 2$. It is the simplest example of a singular RV and appears often in signal theory. Indeed, it describes the values of an autoregressive signal of order one [AR(1)] generated by one of the simplest white noises. This shows that singular RVs are not at all exceptional. This point has already been noted in [6] and [7].

The use of discrete-valued signals is very common in communication problems. For example, in binary communications, the input takes only two values, and if some conditions on the channel are fulfilled, the transmitted signal can be singular. In

Manuscript received June 27, 2003; revised February 28, 2004. This paper was presented in part at the International Conference on Acoustics, Speech, and Signal Processing, Montréal, QC, Canada, May 2004. The associate editor coordinating the review of this paper and approving it for publication was Dr. Franz Hlawatsch.

B. Picinbono is with the Laboratoire des Signaux et Systèmes (L2S) (a joint laboratory of the C.N.R.S. and the École Supérieure d'Électricité), University of Paris-Orsay, 91192, Gif sur Yvette, France (e-mail: bernard.picinbono@1ss.supelec.fr).

J.-Y. Tourneret is with ENSEEIHT/IRIT/TÉSA, 31071 Toulouse cedex 7, France (e-mail: jean-yves.tourneret@tesa.prd.fr).

Digital Object Identifier 10.1109/TSP.2004.840783 this case, the calculation of the probability of error requires specific attention [7].

In this paper, we will show that singularity of autoregressive moving average (ARMA) signals is the result of a combination of two points: discrete-valued inputs and some properties of the poles of the system. More precisely, we will show that singularity results from the pole location inside a circle called the circle of singularity. It is the cornerstone of the discussion of singularity, as is the unit circle for stability. After introducing the basic theory of the problem, we will present various computer simulations in order to highlight a phenomenon widely ignored up to now.

\section{THEORETICAL APPROACHES}

\section{A. Singularity}

Let $w_{k}$ be a discrete-valued WN or a sequence of IID RVs $w_{k}$ taking $q$ distinct possible values. The simplest example is the BWN, where $q=2$. Applying this noise at the input of a causal filter of impulse response (IR) $h_{k}$, we obtain the output

$$
x_{k}=\sum_{l \geq 0} h_{l} w_{k-l} .
$$

In the case of an infinite IR filter, we assume that

$$
S=\sum_{k=0}^{\infty}\left|h_{k}\right|<\infty
$$

which means that the filter defined by $h_{k}$ is stable.

The point that the input $\mathrm{WN}$ is discrete implies the so-called purity theorem [2, p. 64]. It states that the RV $x_{k}$ defined by (1) is either continuous, which means that its DF is absolutely continuous, or discrete, which means that its DF is a step function, or singular, as defined at the beginning.

Consider the exponential causal filter for which $h_{k}=a^{k}$. It is related to the recursion $x_{k}=a x_{k-1}+w_{k}$ defining an $\mathrm{AR}(1)$ signal. If $w_{k}$ is an SBWN, we have the following results [2], [8]: If $0<a<1 / 2, x$ is singular, if $a=1 / 2, x$ is uniformly distributed in the interval $[-2,+2]$, and for almost all values of $a$ satisfying $1 / 2<a<1, x$ is continuous. Note that there exist some values of $a, 1 / 2<a<1$ such that $x$ is singular [8].

Our aim is to extend this kind of result to more general discrete-valued WNs $w_{k}$ or filters $h_{k}$ and, especially, to dynamical filters defined by their poles and zeros and introducing ARMA signals.

For this purpose, let us first recall some notations introduced in [2]. The spectrum $S_{F_{x}}$ of the DF $F_{x}(\xi)$ of the $\mathrm{RV} x$ is the set of all the points of variation of this function, and $L\left(S_{F_{x}}\right)$ its the Lebesgue measure. If $L\left(S_{F_{x}}\right)=0$, the RV $x$ cannot be continuous and is thus either discrete or singular. However, it can be shown by a reasoning beyond the scope of this paper [2, p. 66] 
that it cannot be discrete. Thus, the singularity is characterized by the relation $L\left(S_{F_{x}}\right)=0$.

In order to study this measure, it is appropriate to introduce the quantity

$$
\rho_{n}=\sum_{k=n}^{\infty}\left|h_{k}\right| .
$$

The most common result to characterize a singularity is the following one.

Theorem 1: If the input $w_{k}$ is an SBWN and if $\left|h_{n}\right|>\rho_{n+1}$ for all $n \geq 0$, then

$$
L\left(S_{F_{x}}\right)=4 \lim _{n \rightarrow \infty} 2^{n}\left|h_{n}\right| .
$$

It is given without proof in [2, p. 66]. Let us present some consequences of this theorem.

1) As $\left|h_{n}\right|=\rho_{n}-\rho_{n+1}$, the condition $\left|h_{n}\right|>\rho_{n+1}$ can also be written as $\rho_{n}>2 \rho_{n+1}, n \geq 0$.

2) As $\rho_{0}=S$ and $\rho_{1}=S-\left|h_{0}\right|$, this implies that $S>$ $2\left(S-\left|h_{0}\right|\right)$ or $S<2\left|h_{0}\right|$. For the IR $h_{k}=a^{k}$, we have $h_{0}=1$ and $S=1 /(1-a)$. Thus, the condition $S<2\left|h_{0}\right|$ yields $a<1 / 2$. It is easy to verify that this implies $\rho_{n}>2 \rho_{n+1}, \forall n>0$. Therefore, (4) can be applied and, because $a<1 / 2$, this yields $L\left(S_{F_{x}}\right)=0$ and the singularity of $x$.

However, this theorem is often insufficient to ensure singularity. First, it is valid only for SBWN, which is a strong limitation. Second, it introduces in many cases a domain of singularity that is too small. Third, for ARMA signals discussed in this paper, it is often impossible to calculate explicitly $\rho_{n}$ of (3). Therefore, a more general condition is required, and for this, instead of having the exact value of $L\left(S_{F_{x}}\right)$, it is sufficient to calculate an upper bound of this measure. This is the purpose of the following proposition.

Theorem 2: Let $q$ be the number of distinct possible values of the discrete IID random variables $w_{k}$. If the filter with impulse response $g_{k}=q^{k} h_{k}$ is stable, which means that $\sum_{k=0}^{\infty}\left|g_{k}\right|<$ $\infty$, then the random variable $x=\sum_{k=0}^{\infty} h_{k} w_{k}$ is singular.

Proof: Let $A$ be the greatest possible value of $\left|w_{k}\right|$. The random partial sum $\sum_{k=0}^{n-1} h_{k} w_{k}$ takes at the maximum $q^{n}$ distinct values $v_{i}^{n}$. The possible values of the RV $x$ are $v_{i}^{n}+\sum_{k=n}^{\infty} h_{k} \eta_{k}$, where $\eta_{k}$ is one of the possible values of $w_{k}$. They belong to an interval $I_{i}^{n}$ of measure smaller than $2 A \rho_{n}$. As there are $q^{n}$ intervals $I_{i}^{n}$ at the maximum, the possible values of $x$ belong to an interval $I_{n}$ of measure smaller than $2 A q^{n} \rho_{n}$. Outside this interval, the DF $F(\xi)$ of $x$ cannot vary. Thus, $L\left(S_{F_{x}}\right) \leq 2 A q^{n} \rho_{n}$. As this is valid for any $n, L\left(S_{F_{x}}\right)$ satisfies $L\left(S_{F_{x}}\right) \leq 2 A \lim _{n \rightarrow \infty} q^{n} \rho_{n}$. However, as $q>1$, $q^{n} \rho_{n}<\bar{\rho}_{n}$, where $\bar{\rho}_{n}$ is the rest of the series of the general term $q^{k}\left|h_{k}\right|$. This series is convergent because of the stability assumption. Thus, the rest of it tends to $0, \lim _{n \rightarrow \infty} q^{n} \rho_{n}=0$, and $L\left(S_{F_{x}}\right)=0$, which yields the singularity of $x_{k}$. Let us present some comments and consequences of this theorem.

1) Consider again the case of the IR $h_{k}=a^{k}$. The condition of Theorem 2 is satisfied if $a<1 / q$. For $q=2$, we obtain, as with Theorem $1, a<1 / 2$, but this is even valid for a nonsymmetric BWN.
2) In our simulations, we will consider the filter with the IR $h_{k}=(k+1) a^{k}$. Simple algebra yields $\rho_{n}=a^{n}(\alpha+n \beta)$, where $\alpha$ and $\beta$ are constants. Therefore, $L\left(S_{F_{x}}\right)=0$ as soon as $a<1 / q$ or $a<1 / 2$ for $q=2$. Note that Theorem 1 would require the more restrictive condition $a<1-2^{-1 / 2} \approx 0.2929$.

3) Consider now the case of real filters with complex poles, which is especially important in signal processing, as, for example, the filter defined by $h_{k}=a^{k} \sin (k \phi)$ with the two poles $a \exp ( \pm j \phi)$. The sufficient condition for singularity given by Theorem 2 is $|a|<1 / q$. It is the same as for the exponential filter $h_{k}=a^{k}$. On the other hand, it is difficult to calculate $\rho_{n}$ given by (3).

4) It is obvious that Theorem 2 , which requires only the knowledge of the poles of the filter, is much more adapted to ARMA signals than Theorem 1. Indeed, the latter requires the calculation of the sums $\rho_{n}$ from the poles of the IR, which is often a very complicated task. However, there is no hierarchy between the two theorems. There are a few examples where the conditions of validity of Theorem 1 are less restrictive than those of Theorem 2.

5) Circle of singularity: Consider an ARMA signal $x_{k}$ generated by a $q$-valued white noise $w_{k}$ filtered by a stable dynamical filter, which means that all the poles of its transfer function (TF) $H(z)$ are located inside the unit circle called the circle of stability. Consider now the filter with IR $g_{k}=q^{k} h_{k}$. Its TF is obviously $G(z)=H(z / q)$. Thus, with any pole $\pi_{i}$ of $H(z)$, one can associate the pole $q \pi_{i}$ of $G(z)$. The stability condition of Theorem 2 is ensured if $q\left|\pi_{i}\right|<1$ or if all the poles of $H(z)$ are inside the circle with center 0 and radius $1 / q$. By analogy, this circle is called the circle of singularity.

However, note that while the location of the poles inside the unit circle is a necessary and sufficient condition of stability, the location of these poles inside the circle of singularity is only a sufficient condition ensuring singularity. Note also that Theorem 2 is an intrinsic theorem. This means that it is independent of the possible values of the IID RVs $w_{k}$ and of their probabilities but depends only on the number $q$ of their possible values.

\section{B. Fractal Properties}

Consider the random partial sum $x_{[n]}=\sum_{k=0}^{n-1} h_{k} w_{k}$, where $w_{k}$ is an SBWN. This sum takes $2^{n}$ values $v_{i}^{n}$ defined by $v_{i}^{n}=$ $\sum_{k=0}^{n-1} h_{k} \epsilon_{k}$, where $\epsilon_{k}= \pm 1$. Furthermore, $x_{[n]}$ is symmetric because to each value $v_{i}^{n}$, it is possible to associate the value $-v_{i}^{n}$ by simply changing the signs of each $\epsilon_{k}$. Finally, we can assume in all this section that $h_{k}>0$. Indeed, as $\epsilon_{k}= \pm 1$, the possible values $v_{i}^{n}$ are the same if we replace $h_{k}$ by $\left|h_{k}\right|$.

To each value $v_{i}^{n}$, it is possible to associate two values $v_{i}^{n+1}$ defined by $v_{i}^{n} \pm h_{n}$. By repeating this procedure, we can construct a tree where its nodes are the positive values $v_{i}^{n}$ of the RV $x_{[n]}$, as represented in Fig. 1. In this figure, we have only presented the nodes generated by $h_{0}$. There is, of course, a symmetric tree starting from $-h_{0}$. Let us present the consequences on this tree of the conditions of Theorem 1. The fundamental point is that the relation $h_{n}>\rho_{n+1}$ implies that there is $n o$ crossing of the branches of the tree. 


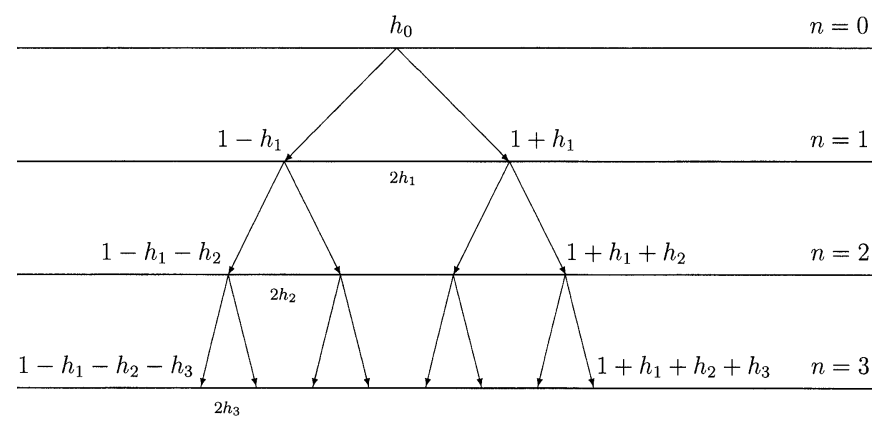

Fig. 1. Tree of successive possible values $v_{i}^{n}$.

Consider first the branches starting from $\pm h_{0}$. There is no crossing if the nodes generated by $h_{0}$ are all positive, which implies by symmetry that the nodes generated by $-h_{0}$ are all negative. This is realized if $h_{0}-\rho_{1}>0$, which is the condition of Theorem 1 for $n=0$. The same procedure can be applied starting from any node $v_{i}^{n}$ of the tree. There is no crossing of branches of the tree coming from an arbitrary node $v_{i}^{n}$ if $v_{i}^{n}$ $h_{n}+\rho_{n+1}<v_{i}^{n}+h_{n}-\rho_{n+1}$, which yields the condition $h_{n}>\rho_{n+1}$ of Theorem 1 .

However, singularity does not require a noncrossing property. To verify this point, consider again the IR $h_{k}=a^{k} \sin (k \phi)$, and suppose that $\phi$ is chosen in such a way that there no $k$ such that $h_{k}=0$. In the tree of Fig. 1, consider an arbitrary node $v_{k-1}^{i}$ and the branches starting from this node. There is crossing of these branches at the step $k+1$ if $\left|h_{k+1}\right|>\left|h_{k}\right|$ or

$$
a>c_{k} \triangleq \frac{|\sin (k \phi)|}{|\sin [(k+1) \phi]|} .
$$

In order to avoid any crossing of this type in the tree, we must have $a<c_{k}$ for any $k$. This condition can for some values of $\phi$ introduce a value of $a$ much smaller than $a=1 / 2$, ensuring the singularity. For example, for $\phi=0.4950$, the noncrossing property requires $a<0.03$. This means that for all the values satisfying $0.03<a<0.5$, there is simultaneously singularity and crossing. As the conditions of Theorem 1 imply noncrossing property, we deduce that Theorem 1 cannot be used for these values of $a$. This clearly shows that the noncrossing property is not a necessary condition of singularity.

Note, finally, that the noncrossing property is directly connected with the construction of Cantor sets, and this means that Cantor-types distributions [7] are not at all the only distributions introducing singularity.

It results from the structure of the tree that each node generates a local symmetry. Similarly, there are holes, or domains, where the DF $F_{x}(\xi)$ remains constant. One can show that there are an infinite number of such disjoint domains and that their union is equal to the measure of the interval, where $F_{x}(\xi)$ cannot vary. This is the opposite part of the fact that the points of variation of $F_{x}(\xi)$ are a set of zero measure, which is the origin of the singularity.

Note, finally, that the noncrossing property can be valid only for sufficiently large $n$ or if $n \geq N$. The properties of symmetries and holes are also valid with this condition. However, it is no longer possible to apply Theorem 1 , which requires $N=0$.

\section{Hausdorff Dimension}

The Hausdorff dimension (HD) is an appropriate tool in the problems of fractal geometry [10]. Let us show that the previous results on the circle of singularity can be retrieved by using HD. Let us note $d_{H}\left(S_{F_{x}}\right)$ as the HD of the set $S_{F_{x}}$. The HD is connected to the singularity of $x$ by the following results.

- When $d_{H}\left(S_{F_{x}}\right)<1$, the set $S_{F_{x}}$ is totally disconnected [10, p. 30]. Consequently, since $x$ cannot be a purely discrete $\mathrm{RV}$, it is a purely singular RV.

- When $d_{H}\left(S_{F_{x}}\right) \geq 1$, we cannot in general conclude since there are singular RVs $x$ such that $d_{H}\left(S_{F_{x}}\right) \geq 1$.

These remarks show that $d_{H}\left(S_{F_{x}}\right)$ is an imperfect measure of the singularity of $x$. There are some cases where $d_{H}\left(S_{F_{x}}\right)$ can be calculated. This appears especially for the exponential filter with $0<a<1 / 2$ driven by an SBWN. In this case, we have $d_{H}\left(S_{F_{x}}\right)=\ln 2 / \ln (1 / a)<1$, which shows the singularity of $x$ for $0<a<1 / 2$. The computation of $d_{H}\left(S_{F_{x}}\right)$ is often difficult. For instance, the computation of $d_{H}\left(S_{F_{x}}\right)$ for any $a \in$ $[1 / 2,1]$ in the case of the exponential filter driven by an SBWN is still an open problem. However, it is sometimes easy to find an upper bound of $d_{H}\left(S_{F_{x}}\right)$, as shown by the following theorem [10, p. 42].

Theorem 3: If, for any $\delta, S_{F_{x}}$ can be covered by a finite number $n(\delta)$ of intervals $I_{k}$ with diameters $\left|I_{k}\right|$ satisfying $\left|I_{k}\right| \leq \delta$, then $d_{H}\left(S_{F_{x}}\right) \leq B$, where the upper bound $B$ is

$$
B=-\lim _{\delta \rightarrow 0} \frac{\ln [n(\delta)]}{\ln (\delta)} .
$$

This bound is sometimes called the box-counting dimension of $S_{F_{x}}$ [10, p. 38]. Let us apply this result when the conditions of Theorem 2 are satisfied. In this case, $S_{F_{x}}$ can be covered by $q^{n}$ intervals $I_{k}^{n}$ of diameters satisfying $\left|I_{k}^{n}\right|<\delta_{n}=2 A \rho_{n}$, where the same notations as in the proof of Theorem 2 are used. As a consequence, $B=\lim _{n \rightarrow \infty} T_{n}$, with

$$
T_{n}=-\frac{\ln \left(q^{n}\right)}{\ln \left(2 A \rho_{n}\right)}=-\ln q \frac{n}{\ln (2 A)+\ln \rho_{n}} .
$$

This yields $B=-\ln q \lim _{n \rightarrow \infty}\left[n / \ln \rho_{n}\right]$. Let $a$ be the maximum value of $\left|\pi_{i}\right|$, where $\left\{\pi_{i}\right\}$ denotes all the poles of the ARMA filter. It is easy to verify that $\lim \left[n / \ln \rho_{n}\right]=1 / \ln a$, which leads to

$$
B=\frac{\ln q}{\ln \left(\frac{1}{a}\right)}
$$

where $a<1$. The assumptions of Theorem 2 imply that $q<$ $1 / a$, i.e., $B<1$. Thus, the RV $x$ is singular. To summarize, the Hausdorff dimension gives, in another way, a proof of the singularity of $x$ when the assumptions of Theorem 2 are satisfied.

\section{Simulations With AR(1) Signals}

Consider an AR(1) $x_{k}$ signal generated by a SBWN, and let us study its DF. The appropriate tool for this purpose is the histogram. When the DF has a derivative, the normalized histogram yields an evaluation of the PDF. However, as we are dealing with 

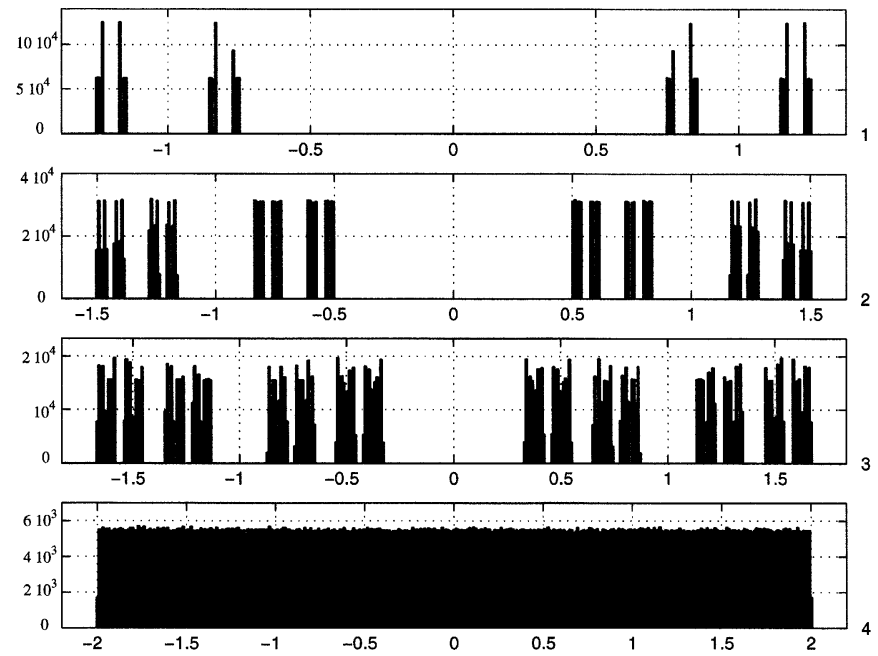

Fig. 2. Histograms of $x_{k}$ for various $a$. 1) $a=0.2$.2) $a=1 / 3$. 3) $a=0.4$. 4) $a=0.5$.

a DF that can be without a PDF, we are obliged to calculate histograms at different scales according to a procedure described below.

To obtain a first picture of the influence of the regression coefficient $a$, various histograms calculated with $2.5 \times 10^{6}$ samples $x_{k}$ are presented in Fig. 2. The values of $x_{k}$ are classified in 400 adjacent cells of the same length covering an interval $[-B,+B]$, where $B=1.1 S$, where $S$ is the sum defined by (2).

Histogram 2.1 shows only a small number of apparent symmetries, while they appear more clearly in the other histograms. Finally, the last histogram corresponds to the theory that predicts, for $a=1 / 2$, a uniform distribution in the interval $[-2,+2]$. Note that the sums $S$ defined by (2) appear clearly as the maximum extension of the histograms. Indeed, the maximum value of $v_{i}^{n}$ is $S$, and the exact values of $S$ are 1.25, 1.5, 1.667, and 2, in complete agreement with Fig. 2.

In order to obtain a better understanding of the singularity, we will analyze, at different scales, histogram 2 of Fig. 2 corresponding to $a=1 / 3$. This value appears in the definition to the ternary Cantor set [2, p. 8]. For highlighting the symmetries described in the previous section, the center of each histogram of Fig. 3 is chosen at one of the symmetry centers already introduced. Furthermore, for illustrating the fractal autosimilarity, the intervals of analysis of each histogram correspond to the domain of symmetry previously introduced. Finally, each histogram contains 400 cells.

The results are presented in Fig. 3, which follows 2) of Fig. 2. The center of symmetries calculated for $a=1 / 3$ are $0,1,1.333$, $1.444,1.4815,1.4938$, and 1.4979. The six histograms of Fig. 3 correspond to the analysis of $2.6 \times 10^{6}$ successive samples of the signal $x_{k}$. The phenomenon of autosimilarity is remarkable. In order to appreciate the precision of the result, note that histogram 6 of Fig. 3 corresponds to the interval $[1.4958,1.5]$ of histogram 2 of Fig. 2. Thus, in spite of a scaling of the order of $3^{6}=729$, the structure of the histograms remains almost the same. This corresponds perfectly to the process of generation by the tree of Fig. 1 and explains the origin of the singularity of the signal. Indeed, this figure can be realized for any node $v_{i}^{n}$ of the tree and passing to the limit $n \rightarrow \infty$, where we see that any

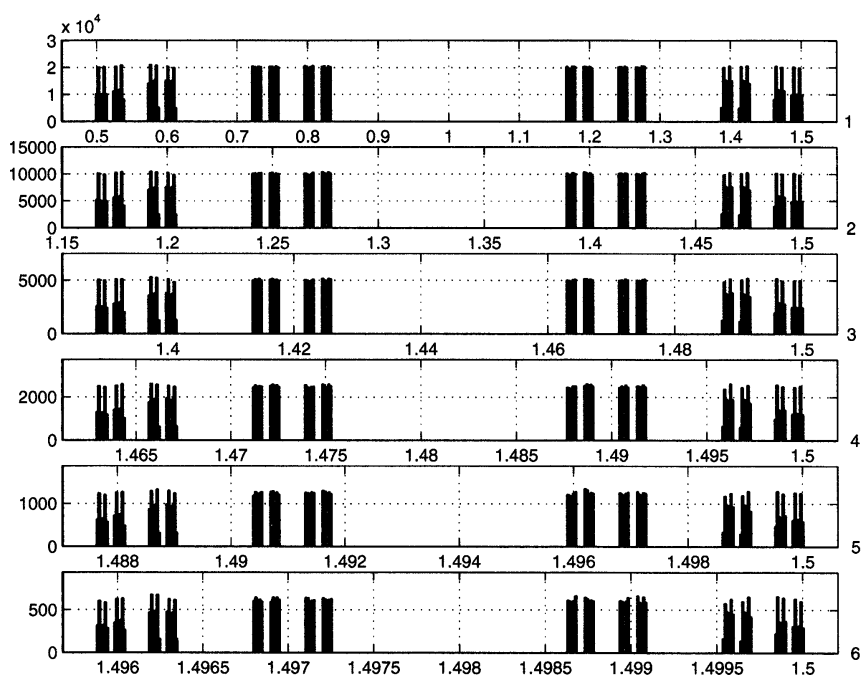

Fig. 3. Histograms of $x_{k}$ at different scales, $a=1 / 3$.

interval $\Delta \xi$ does not contain points of variation of the DF $F(\xi)$, which means that the derivative is almost everywhere equal to zero or that $x_{n}$ is singular.

\section{AR Signals With Multiple Poles}

The AR(1) signals analyzed in the previous section are generated from $w_{k}$ by the filter $(1)$ with the transfer function $H_{1}(z)=$ $z /(z-a)$. In order to better understand the phenomenon of singularity of signals, we will now study AR signals generated by filters with transfer functions $H_{n}(z)=\left[H_{1}(z)\right]^{n}$, introducing a pole of order $n$ in $a$. For the sake of simplicity, we restrict our analysis to the case where $n=3$. The IR of this filter is $(1 / 2) k(k+1) a^{k}$, and the sum $S$ defined by (2) is $(1-a)^{-3}$. Various histograms at different scales are given in Figs. 4 and 5. These figures are calculated with $a=0.4$ and $a=0.2$. The correponding values of $S$ previously calculated are indicated.

For $a=0.4$, the fractal structure with a hole in the center of the histogram begins to appear with histogram 5, which corrresponds to a reduction of approximately 80 times of the interval of histogram 1 . Note that the interval of analysis of the last histogram is 1450 times smaller than the first of the same figure. This shows that with a signal and a triple pole and for this value of $a$, the singularity requires analysis at a very large scale of reduction of the interval of variations of the DF.

The behavior of AR signals with multiple poles requires some additional comments. It was noted in the comments of Theorem 2 that the application of the conditions of Theorem 1 requires $S<2 h_{0}=2$. This yields $a<1-2^{-1 / 3} \approx 0.2063$, which is not satisfied for $a=0.4$ used in Fig. 4. In order to appreciate the difference appearing when this is satisfied, the same histograms calculated for $a=0.2$ are presented in Fig. 5. The condition of noncrossing of the branches of the tree is satisfied, and this appears clearly on the histograms. The difference is that there is no regular repetition of the same structure, as in the case of the pure exponential impulse response of $\mathrm{AR}(1)$ signals analyzed in Fig. 3. However, this regularity tends to appear for large values of $n$ because in this case, the term $a^{k}$ is dominant in the IR. Note also that the hole centered at zero is very small in Fig. 5 because its existence requires $a>0.2063$, which is the limit 


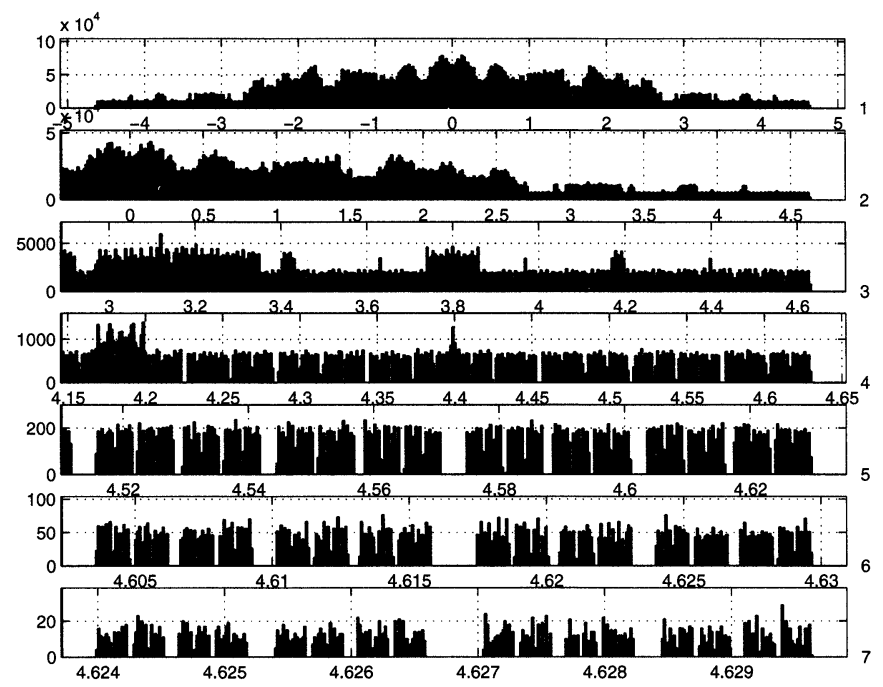

Fig. 4. Histograms for triple pole at different scales $a=0.4, S=4.6296$.

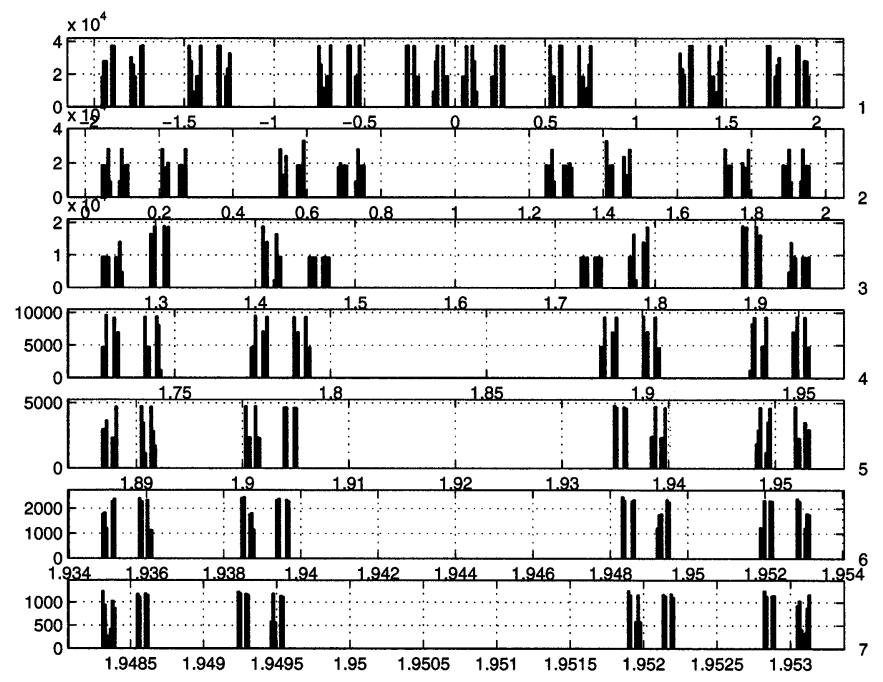

Fig. 5. Histograms for triple pole at different scales $a=0.2, S=1.9531$.

of the validity of Theorem 1 , and this limit is very close to 0.2 , which is the value of $a$ used in the simulation.

\section{ARMA SIGNALS}

Up to now, we have only considered AR signals. In order to verify that the same situation occurs with ARMA models, we present a simulation corresponding to an $\operatorname{ARMA}(2,2)$ signal in the particular case of a double pole and zero. Thus, the TF of the filter is

$$
H(z)=\frac{(z+b)^{2}}{(z-a)^{2}}
$$

which yields the impulse response

$$
\begin{aligned}
h_{k} & =(k+1) a^{k}+2 b k a^{k-1}+b^{2}(k+1) a^{k-2} \\
h(0) & =1, \quad h_{1}=2(a+b) .
\end{aligned}
$$

The use of this IR to analyze the singularity from the conditions of Theorem 1 is complicated. On the other hand, according to Theorem 2, singularity is ensured as soon as $a<0.5$.

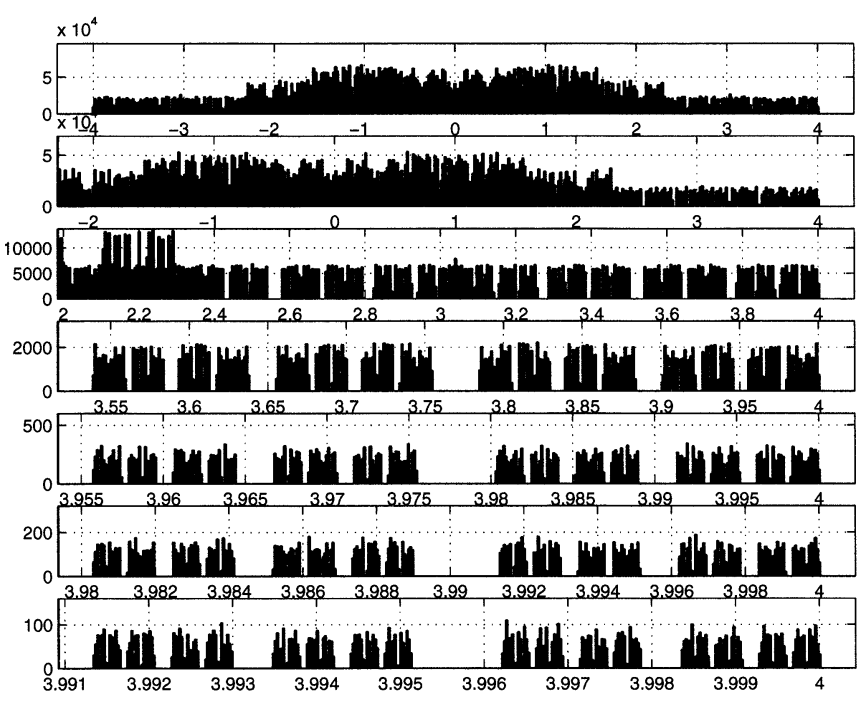

Fig. 6. Histograms for ARMA signal at different scales $a=0.4, b=0.2$, $S=4$.

The results calculated with $1.2 \times 10^{7}$ samples and corresponding to the values $a=0.4$ and $b=0.2$ of the parameters of the model are presented in Fig. 6. For these values, $S=\sum h_{k}=H(1)=4$, which appears clearly in the figure. The scaling between the first and the last histograms is of the order of 950. The first three histograms show a strong crossing phenomenon, which means that the conditions of Theorem 1 are not satisfied. However, the fractal structure leading to the singularity begins to appear in the last histograms of this figure.

\section{CONCLUSION}

This paper has introduced a new class of signals called singular random signals. They are characterized by the fact that the distribution functions of their values at each time instant are singular or continuous but without derivative. This implies that there is neither probability density function nor discrete mass of probability. Usually considered to be only of mathematical interest without impact on practical problems, we have shown that singular distributions appear, to the contrary, to describe a large class of simple signals. They are ARMA signals generated from discrete-valued white noise and filters with poles located inside the circle of singularity defined in the analysis. Therefore, singularity can appear as soon as the input generating an ARMA signal is discrete-valued, as in many communication systems. The origin of singularity and its relation with fractal structures have been analyzed. Various simulations concerning AR or ARMA signals have been presented. The results are in good accordance with theoretical analysis and show that singularity is not at totally a mathematical curiosity but can appear in very simple situations.

Many open questions remain for future work. Among the points to be clarified, we can indicate, for example, the role of correlated inputs on the singularity of the output. Another point to clarify is the consequences of the absence of a probability density function in all the statistical signal processing methods where this density is used, as, for example, those using likelihood functions. 


\section{REFERENCES}

[1] E. Wong and B. Hajek, Stochastic Processes in Engineering Systems. New-York: Springer-Verlag, 1985.

[2] E. Lukacs, Characteristic Functions. London, U.K.: Griffin, 1970.

[3] A. Papoulis, Probability, Random Variables and Stochastic Processes. New York: McGraw Hill, 1984.

[4] C. W. Helstrom, Probability and Stochastic Processes for Engineers. New York: Macmillan, 1991.

[5] B. Picinbono, Random Signals and Systems. Englewood Cliffs, NJ: Prentice-Hall, 1993.

[6] J.-Y. Tourneret and B. Lacaze, "Etude et simulation des lois de probabilité de sorties de modèles paramétriques," Traitement du signal, vol. 11, pp. 271-281, 1994.

[7] P. H. Wittke, W. S. Smith, and L. L. Campbell, "Infinite series of interferences variables with Cantor-type distributions," IEEE Trans. Inf. Theory, vol. 34, pp. 1428-1436, Nov. 1988.

[8] Y. Peres, W. Schlag, and B. Solomyak, "Sixty years of Bernoulli convolutions, fractals and stochastics II," in Progress in Probability, C. Bt, S. Graf, and M. Zähle, Eds. Boston, MA: Birkhäuser, 2000, vol. 46, pp. 95-106.

[9] Y. Peres and B. Solomyak, "Self-similar measures and intersections of Cantor sets," Trans. Amer. Math. Soc., vol. 350, pp. 4065-4087, 1998.

[10] K. Falconer, Fractal Geometry. Mathematical Foundations and Applications. Chichester, U.K.: Wiley, 1990.

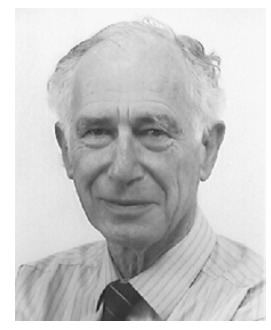

Bernard Picinbono (M'63-SM'79-F'85) was born in Algiers, Algeria, on March 21, 1933. He received the Agrégation des Sciences Physiques degree in 1956 and the Doctorat détat degree in 1960.

From 1960 to 1965, he was a Professor with the Department of Physics, University of Algiers, and in 1965, he joined the University of Paris-Sud, Orsay, France, to which he is still attached (Emeritus). He was the President of this university between 1971 and 1975 and Director of the Laboratoire des Signaux et Systémes (L25), which is a joint research center of the Centre National de Ia Recherche Scientifique (CNRS), the École Supdrieure d'Electricité (ESE), and the Université de Paris-Sud (UPS) between 1975 and 1987. Between 1990 and 1995, he was General Director of the ESE. His research interests include statistical signal processing, detection and estimation, and stochastic processes. He is the author of numerous papers and books on these subjects.

Dr. Picinbono is a member of the French Academy of Sciences and of the Académie des Technologies. He is President of the GRETSI Conference organized every two years in France and devoted to Signal and Image Processing problems. He was Co-Chairman of the 1982 IEEE International Symposium of Information Theory, Les Arcs, France.

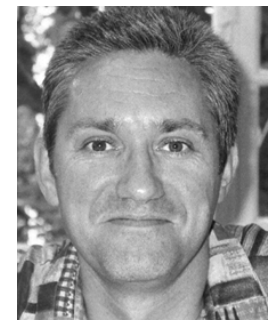

Jean-Yves Tourneret received the ingénieur degree in electrical engineering in 1989 from Ecole Nationale Supérieure d'Electronique, d'Electrotechnique, d'Informatique, et d'Hydraulique (ENSEEIHT), Toulouse, France, and the Ph.D. degree from the National Polytechnic Institute, Toulouse, in 1992.

$\mathrm{He}$ is currently a professor with the ENSEEIHT. $\mathrm{He}$ is a member of the IRIT Laboratory (UMR 5505 of the CNRS), where his research activity is centered around estimation, detection, and classification of non-Gaussian and non-Stationnary processes.

Dr. Tourneret was the program chair of the European conference on signal processing (EUSIPCO), Toulouse, in 2002. He is also a member of different technical committes including the Signal Processing Theory and Methods (SPTM) committee of the IEEE Signal Processing Society. 